\title{
Asymptotic Behavior of Critical Infection Rates for Threshold-one Contact Processes on Lattices and Regular Trees
}

\author{
Xiaofeng Xue * \\ Peking University
}

\begin{abstract}
In this paper we study threshold-one contact processes on lattices and regular trees. The asymptotic behavior of the critical infection rates as the degrees of the graphs growing to infinity are obtained. Defining $\lambda_{c}$ as the supremum of infection rates which causes extinction of the process at equilibrium, we prove that $n \lambda_{c}^{\mathbf{T}^{n}} \rightarrow 1$ and $2 d \lambda_{c}^{\mathbf{Z}^{d}} \rightarrow 1$ as $n, d \rightarrow+\infty$. Our result is a development of the conclusion that $\lambda_{c}^{\mathbf{Z}^{d}} \leq \frac{2.18}{d}$ shown in 2. To prove our main result, a crucial lemma about the probability of a simple random walk on a lattice returning to zero is obtained. In details, the lemma is that $\lim _{d \rightarrow+\infty} 2 d P\left(\exists n \geq 1, S_{n}^{(d)}=0\right)=1$, where $S_{n}^{(d)}$ is a simple random walk on $\mathbf{Z}^{d}$ with $S_{0}^{(d)}=0$.
\end{abstract}

Keywords: contact process, threshold-one, critical value, asymptotic behavior.

\section{Introduction}

In this paper we study threshold-one contact processes on lattices and regular trees. For a graph $G$, threshold-one contact process on $G$ is with state space $\{0,1\}^{G}$, which means that at each vertex on $G$, there is a spin taking value 0 or 1 . For each $x \in G$ and any configuration $\eta \in\{0,1\}^{G}$, we denote by $\eta(x)$ the value of $x$. For any $t>0$, the configuration of the process at $t$ is denoted by $\eta_{t}$.

*E-mail: masonxuexf@math.pku.edu.cn Address: School of Mathematical Sciences, Peking University, Beijing 100871, China. 
For each $x \in G$ and $t>0$, we define

$$
\eta_{t-}(x)=\lim _{s<t, s \uparrow t} \eta_{s}(x)
$$

as the value of $x$ at the moment just before $t$. For any $x, y \in G$, we say that they are neighbors if there is an edge connecting them, denoted by $x \sim y$.

Now we explain how the process evolves. At the beginning, each vertex takes 0 or 1 according to some probability distribution. Then, the process evolves depending on independent Poisson processes $\left\{N_{x}(t)\right\}_{x \in G}$ and $\left\{Y_{x}(t)\right\}_{x \in G}$. For each vertex $x \in G, N_{x}(\cdot)$ is with rate one while $Y_{x}(\cdot)$ is with rate $\lambda>0 . \lambda$ is called the infection rate. The value of $x$ may flip only at the event times of $N_{x}(\cdot)$ and $Y_{x}(\cdot)$. For any event time $s$ of $N_{x}(\cdot), \eta_{s}(x)$ takes 0 no matter whatever $\eta_{s-}(x)$ is. For any event time $r$ of $Y_{x}(\cdot), \eta_{r}(x)$ does not flip when $\eta_{r-}(x)=1$. When $\eta_{r-}(x)=0, \eta_{r}(x)$ flips to 1 at $r$ if and only if there exists a neighbor $y$ of $x$ such that $\eta_{r-}(y)=1$. Therefore the threshold-one contact process $\eta_{t}$ is a spin system (See the definition of spin systems in Chapter 3 of [‥) with flip rates given by

$$
c(x, \eta)= \begin{cases}1 \quad \text { if } \quad \eta(x)=1, \\ \lambda \quad \text { if } \quad \eta(x)=0 \quad \text { and } \quad \sum_{y: y \sim x} \eta(y)>0, \\ 0 \quad \text { otherwise }\end{cases}
$$

for any configuration $\eta \in\{0,1\}^{G}$.

Intuitively, the process describes the spread of an infection disease on a network. Each $x \in G$ stands for an individual who may be infected by the disease. 1 and 0 represent the state 'infected' and 'healthy' respectively. An individual in the infected state will wait for an exponential time with rate one to be healed. An healthy individual will wait for an exponential time with rate $\lambda$ to be infected if and only if there is at least one neighbor of it is in the infected state.

In later sections, we write $\eta_{t}$ as $\eta_{t}^{\eta}$ when $\eta_{0}=\eta \in\{0,1\}^{G}$. We denote by $\delta_{1}$ the configuration that all vertices take value 1 . Since the threshold-one contact process is attractive (See the definition of 'attractive' in Chapter 3 of [8].), it is easy to see that

$$
P\left(\eta_{t}^{\delta_{1}}(x)=1\right)
$$

decreases with $t$ for each $x \in G$. Hence it is reasonable to define

$$
\mu(x)=\lim _{t \rightarrow+\infty} P\left(\eta_{t}^{\delta_{1}}(x)=1\right)
$$


for each $x \in G$. To distinguish processes on different graphs with different infection rates, we write $\mu$ as $\mu_{\lambda}^{G}$. According to the basic coupling of spin systems (See Chapter 3 of 8 .). , it is easy to see that

$$
\mu_{\lambda_{1}}^{G}(x) \geq \mu_{\lambda_{2}}^{G}(x)
$$

for $\lambda_{1}>\lambda_{2}$. Therefore it is reasonable to define

$$
\lambda_{c}^{G}=\sup \left\{\lambda: \sup _{x \in G} \mu_{\lambda}^{G}(x)=0\right\} .
$$

$\lambda_{c}^{G}$ is called the critical value of the infection rate. According to (1.2), when $\lambda<\lambda_{c}, \eta_{t}$ converges weakly to $\delta_{0}$, the configuration that all vertices take 0 . Hence the disease is extinct when $\lambda<\lambda_{c}$. In this paper, we are concerned with the estimation of $\lambda_{c}^{G}$ for $G$ is a lattice or a regular tree. Our results will be introduced in following sections.

The threshold contact process is introduced in [2] as a tool to study threshold voter model since when infection rate $\lambda=1$, threshold voter models can be bounded below by threshold contact processes (See [1, 2], [5, 9, [10, 14.). In [2], the threshold is considered to be one. It is shown in [2] that thresholdone contact process has an additive dual process. Due to the additivity of the dual process, it is suggested that the threshold-one contact process has similar features with that of linear contact process which is additive and self-dual (See Chapter 6 of [8].). In recent years, more works are concerned on the case that the threshold is bigger than one such as [3], [11] and [14. It is studied in [3] and 14. the critical infection rates and critical density points for threshold contact processes and threshold voter models on regular trees. It is showed in [2] and [1] that the critical infection rate for threshold contact process on lattice converges to 0 as the degree grows to infinity. This paper is a development of this result in the case of threshold one, as we give the asymptotic behavior of the critical infection rate.

\section{Main results}

Now we introduce our main results. We obtain the asymptotic behavior of $\lambda_{c}$ for the process on lattice and regular tree as the degree of the graph grows to infinity. In this paper, high-degree lattice with degree $2 d$ is denoted by $\mathbf{Z}^{d}$ 
while regular tree with degree $n+1$ is denoted by $\mathbf{T}^{n}$. The following theorem is our main result.

Theorem 2.1. $\lambda_{c}^{G}$ is defined as that in (1.2), then

$$
\lim _{d \rightarrow+\infty} 2 d \lambda_{c}^{Z^{d}}=1
$$

and

$$
\lim _{n \rightarrow+\infty} n \lambda_{c}^{T^{n}}=1
$$

Theorem 2.1 shows that for lattices and regular trees, when the degree is large, $\lambda_{c}$ is approximate to the reciprocal for the degree. In 2], Cox and Durrett shows that $\lambda_{c}^{\mathbf{Z}^{d}} \leq 2.18 / d$. (2.1) is a development of this result. For classical linear contact process, similar asymptotic behaviors of critical value as (2.1) and (2.2) were proven in [4] and [12. Theorem 2.1 shows that the critical value of threshold-one contact process is with the same asymptotic behavior as that of linear contact process.

It is shown in [11] that $\lim _{d \rightarrow+\infty} \lambda_{c}^{\mathbf{Z}^{d}}(K)=0$ where $\lambda_{c}^{\mathbf{Z}^{d}}(K)$ is the critical value of threshold $K \geq 2$ contact process on $\mathbf{Z}^{d}$. By Theorem 2.1, it is natural to guess that

$$
\lim _{d \rightarrow+\infty} 2 d \lambda_{c}^{\mathbf{Z}^{d}}(K)=K
$$

for $K \geq 2$. But we have no idea whether this conjecture is right.

We divide the proof of Theorem 2.1 into several sections. In Section 3 , we will prove $\liminf \operatorname{int}_{d \rightarrow+\infty} 2 d \lambda_{c}^{\mathbf{Z}^{d}} \geq 1$ and $\liminf _{n \rightarrow+\infty} n \lambda_{c}^{\mathbf{T}^{n}} \geq 1$ by giving a lower bound of $\lambda_{c}$. In Section 4 we will give an upper bound of $\lambda_{c}^{\mathbf{T}^{n}}$ to accomplish the proof of (2.2). In Section 5 we will give an upper bound of $\lambda_{c}^{\mathbf{Z}^{d}}$ to accomplish the proof of (2.1).

\section{Lower bound}

In this section we will give a lower bound of $\lambda_{c}$. To do so, we introduce another stochastic process as a tool, which is denoted by $\xi_{t}$. The state space of $\xi_{t}$ on graph $G$ is $\mathbf{N}^{G}$, where $\mathbf{N}$ is the set of nonnegative integers, which means that at each vertex there is an nonnegative integer. $\xi_{t}$ evolves as following. $\left\{N_{x}(\cdot)\right\}_{x \in G}$ and $\left\{Y_{x}(\cdot)\right\}_{x \in G}$ are Poisson processes as that in the definition of threshold-one contact process. For each $x \in G, \xi(x)$ may change only at event times of $N_{x}(\cdot)$ and $Y_{x}(\cdot)$. At any event time $s$ of $N_{x}(\cdot), \xi_{s}(x)$ takes 0 no matter whatever $\xi_{s-}(x)$ 
is. At any event time $r$ of $Y_{x}(\cdot), \xi(x)$ flips to $\xi_{r}(x)=\xi_{r-}(x)+\sum_{y: y \sim x} \xi_{r-}(y)$ from $\xi_{r-}(x)$. From the definition, it is easy to see that $\xi_{t}$ is a linear model (See Chapter 9 of [8].). As a Markov process, $\xi_{t}$ can also be described via its generator. For any $\xi \in \mathbf{N}^{G}, x \in G$ and $m \in \mathbf{N}$, we define $\xi^{x, m} \in \mathbf{N}^{G}$ as

$$
\xi^{x, m}(y)=\left\{\begin{array}{lll}
\xi(y) & \text { if } & y \neq x, \\
m & \text { if } & y=x .
\end{array}\right.
$$

Then the generator $\Omega$ of $\xi_{t}$ is given by

$$
\begin{aligned}
\Omega f(\xi)= & \sum_{x \in G}\left[f\left(\xi^{x, 0}\right)-f(\xi)\right] \\
& +\sum_{x \in G} \lambda\left[f\left(\xi^{x, \xi(x)+\sum_{y: y \sim x} \xi(y)}\right)-f(\xi)\right]
\end{aligned}
$$

for any $f \in C\left(\mathbf{N}^{G}\right)$ properly fast decaying.

Intuitively the process $\xi_{t}$ counts the (degree of) seriousness of the disease throughout the process. At event times of $Y_{x}(\cdot)$ an infected individual $x$ is able to further infected by its neighbors. Whenever that occurs, we simply add the seriousness of the disease of $x$ by the sum of all the seriousness of the disease of $x$ 's neighbors.

We explain the connection between $\xi_{t}$ and the threshold-one contact process $\eta_{t}$. For each $x \in G$ and $t \geq 0$, let $\widehat{\eta}_{t}(x)=1_{\left\{\xi_{t}(x)>0\right\}}$. We claim that $\widehat{\eta}_{t}$ is threshold-one contact process with flip rates given by (1.1). According to the definition of $\xi_{t}$, at any event time $s$ of $N_{x}(\cdot), \xi_{s}(x)$ takes 0 and hence $\widehat{\eta}_{s}(x)=0$. At any event time $r$ of $Y_{x}(\cdot)$, if $\xi_{r-}(x)>0$, then $\xi_{r}(x) \geq \xi_{r-}(x)>0$ and hence $\widehat{\eta}(x)$ does not flip from 1 at $r$. If $\xi_{r-}(x)=\widehat{\eta}_{r-}(x)=0$, then $\xi_{r}(x)>0$ if and only if there exists a neighbor $y$ of $x$ such that $\xi_{r-}(y)>0$. In other words, $\widehat{\eta}(x)$ flips from 0 to 1 at $r$ if and only if there exists a neighbor $y$ of $x$ such that $\widehat{\eta}_{r-}(y)=1$. Therefore $\widehat{\eta}_{t}$ evolves as a threshold-one contact process. As a result, $\xi_{t}^{\delta_{1}}$ and $\eta_{t}^{\delta_{1}}$ with same infection rate $\lambda$ on $G$ can be coupled such that

$$
\eta_{t}(x)=1_{\left\{\xi_{t}(x)>0\right\}}
$$

for each $x \in G$. By (3.3) and Chebyshev's inequality,

$$
P\left(\eta_{t}^{\delta_{1}}(x)=1\right)=P\left(\xi_{t}^{\delta_{1}}(x) \geq 1\right) \leq E \xi_{t}^{\delta_{1}}(x) .
$$

Now we give a lower bound of $\lambda_{c}^{G}$ where $G$ is a simple regular graph. A regular graph is a graph where each vertex has the same degree, and simple 
graphs are those containing no self-loops or multiple edges. $\mathbf{Z}^{d}$ and $\mathbf{T}^{n}$ are all simple regular graphs. The following theorem gives a lower bound of $\lambda_{c}$.

Theorem 3.1. For a simple regular graph $G$ with degree $r$,

$$
\lambda_{c}^{G} \geq \frac{1}{r} .
$$

The following proposition is a direct corollary of Theorem 3.1

\section{Corollary 3.2.}

$$
\lambda_{c}^{Z^{d}} \geq \frac{1}{2 d} \quad \text { and } \quad \lambda_{c}^{T^{n}} \geq \frac{1}{n+1}
$$

Hence,

$$
\liminf _{d \rightarrow+\infty} 2 d \lambda_{c}^{Z^{d}} \geq 1 \quad \text { and } \quad \liminf _{n \rightarrow+\infty} n \lambda_{c}^{T^{n}} \geq 1 .
$$

Proof of Theorem 3.1. Consider $\xi_{t}$ with infection rate $\lambda$ on $G$. According to the generator $\Omega$ of $\xi_{t}$ given in (3.2), we can prove that

$$
\frac{d}{d t} E \xi_{t}^{\delta_{1}}(x)=-E \xi_{t}^{\delta_{1}}(x)+\lambda \sum_{y: y \sim x} E \xi_{t}^{\delta_{1}}(y)
$$

for each $x \in G$.

Intuitively, (3.6) is with the form $\frac{d}{d t} E f\left(\xi_{t}\right)=E \Omega f\left(\xi_{t}\right)$ as an 'application' of Hille-Yosida Theorem (See Theorem 1.2.9 of [8]). However, the state space $\mathbf{N}^{G}$ of $\xi_{t}$ is not compact, which does not satisfy the condition of Hille-Yosida Theorem. To prove (3.6) rigorously, we need Theorem 1.27 in Chapter 9 of [8]. For more details, see Appendix A.2.

It is easy to verify that

$$
F_{t}(x)=\exp \{t(\lambda r-1)\}
$$

for each $x$ is a solution of ODE (3.6) with $F_{0}=1$. According to classical theory of functional analysis, it is easy to see that ODE (3.6) with initial condition $E \xi_{0}^{\delta_{1}}=\mathbf{1}$ has an unique solution. Therefore,

$$
E \xi_{t}^{\delta_{1}}(x)=F_{t}(x)=\exp \{t(\lambda r-1)\}
$$

for $t \geq 0$. By (3.4), when $\lambda<\frac{1}{r}$,

$$
\mu_{\lambda}^{G}(x)=\lim _{t \rightarrow+\infty} P\left(\eta_{t}^{\delta_{1}}(x)=1\right) \leq \lim _{t \rightarrow+\infty} E \xi_{t}^{\delta_{1}}(x)=\lim _{t \rightarrow+\infty} \exp \{t(\lambda r-1)\}=0
$$

for each $x \in G$, and hence

$$
\lambda_{c}^{G} \geq \frac{1}{r}
$$


Since $\mathbf{Z}^{d}$ is simple regular graph with degree $2 d$ and $\mathbf{T}^{n}$ is simple regular graph with degree $n+1$, Corollary 3.2 follows from Theorem 3.1 directly.

\section{Upper bound: the case of regular trees}

In this section we will give an upper bound of $\lambda_{c}^{\mathbf{T}^{n}}$ and accomplish the proof of (2.2). A dual process $A_{t}$ introduced in [2] is crucial for our approach. The process $A_{t}$ on $\mathbf{T}^{n}$ is with state space

$$
2^{\mathbf{T}^{n}}=\left\{B: B \subseteq \mathbf{T}^{n}\right\}
$$

For each $x \in \mathbf{T}^{n}, N_{x}(\cdot)$ and $Y_{x}(\cdot)$ are Poisson processes as that in the definition of threshold-one contact process $\eta_{t}$. $A_{t}$ evolves as following. For each $x \in \mathbf{T}^{n}$ and any event time $s$ of $N_{x}(\cdot), A_{s}=A_{s-} \backslash\{x\}$. At any event time $r$ of $Y_{x}(\cdot)$, $A_{r}=A_{r-} \cup\{y: y \sim x\}$ if $x \in A_{r-}$, otherwise $A_{r}=A_{r-}$. We write $A_{t}$ as $A_{t}^{A}$ when $A_{0}=A \subseteq \mathbf{T}^{n}$, then it is shown in [2] that

$$
P\left(\eta_{t}^{\delta_{1}}(x)=1\right)=P\left(A_{t}^{\{x\}} \neq \emptyset\right)
$$

for each $x \in \mathbf{T}^{n}$ (See a simple proof of [4.1) in Part Two of [10]).

We introduce a branching process $S_{t} \in 2^{\mathbf{T}^{n}}$ to bound below the growth of $A_{t}$. To introduce $S_{t}, \mathbf{T}^{n}$ is considered as an oriented regular tree that for each $x \in \mathbf{T}^{n}$, one neighbor of $x$ is its 'farther' while the other $n$ neighbors of $x$ are its sons. We denote by $x \rightarrow y$ that $y$ is a son of $x$. $S_{t}$ are evolves as following. For each $x \in \mathbf{T}^{n}$ and any event time $s$ of $N_{x}(\cdot), S_{s}=S_{s-} \backslash\{x\}$. At any event time $r$ of $Y_{x}(\cdot), S_{r}=\left(S_{r-} \cup\{y: x \rightarrow y\}\right) \backslash\{x\}$ if $x \in S_{r-}$, otherwise $S_{r}=S_{r_{-}}$. We write $S_{t}$ as $S_{t}^{A}$ when $S_{0}=A \subseteq \mathbf{T}^{n}$.

By basic coupling, it is easy to see that

$$
A_{t}^{\{x\}} \supseteq S_{t}^{\{x\}}
$$

for each $x \in \mathbf{T}^{n}$ and any $t \geq 0$. Therefore,

$$
P\left(\eta_{t}^{\delta_{1}}(x)=1\right)=P\left(A_{t}^{\{x\}} \neq \emptyset\right) \geq P\left(S_{t}^{\{x\}} \neq \emptyset\right) .
$$

According to the definition of $S_{t}$, for each $x \in S_{t}, x$ will be replaced by $n$ sons with probability $\frac{\lambda}{\lambda+1}$ or be kicked out from $S_{t}$ without 'compensation' with 
probability $\frac{1}{\lambda+1}$. Therefore $S_{t}$ is a branching process with offspring distribution with mean

$$
\frac{n \lambda}{\lambda+1}
$$

The following theorem gives an upper bound of $\lambda_{c}^{\mathbf{T}^{n}}$.

\section{Theorem 4.1.}

$$
\lambda_{c}^{T^{n}} \leq \frac{1}{n-1}
$$

and hence

$$
\limsup _{n \rightarrow+\infty} n \lambda_{c}^{T^{n}} \leq 1
$$

Proof. For $\lambda>\frac{1}{n-1}$,

$$
\frac{\lambda}{\lambda+1} n>1 .
$$

Therefore according to classical theorems of branching process (See Chapter 3 of $[6]$.),

$$
P\left(S_{t}^{\{x\}} \neq \emptyset \text { for any } t \geq 0\right)>0
$$

when $\lambda>\frac{1}{n-1}$. By (4.2),

$$
\begin{aligned}
\mu_{\lambda}^{\mathbf{T}^{n}}(x) & =\lim _{t \rightarrow+\infty} P\left(\eta_{t}^{\delta_{1}}(x)=1\right)=\lim _{t \rightarrow+\infty} P\left(A_{t}^{\{x\}} \neq \emptyset\right) \\
& \geq \lim _{t \rightarrow+\infty} P\left(S_{t}^{\{x\}} \neq \emptyset\right)=P\left(S_{t}^{\{x\}} \neq \emptyset \quad \text { for any } t \geq 0\right) .
\end{aligned}
$$

Therefore, $\mu_{\lambda}^{\mathbf{T}^{n}}(x)>0$ for $\lambda>\frac{1}{n-1}$ and

$$
\lambda_{c}^{\mathbf{T}^{n}} \leq \frac{1}{n-1} .
$$

(2.2) is a direct corollary of Corollary 3.2 and Theorem 4.1. Furthermore, these two theorems show that

$$
\frac{1}{n+1} \leq \lambda_{c}^{\mathbf{T}^{n}} \leq \frac{1}{n-1}
$$

for each $n \geq 1$. 


\section{Upper bound: the case of lattices}

In this section we will give an upper bound of $\lambda_{c}^{\mathbf{Z}^{d}}$ and accomplish the proof of (2.1). The approach in Section 4 fails here because there are many graph-loops on $\mathbf{Z}^{d}$ so that $A_{t}$ can not be bounded below by a branching process. We are inspired a lot by the approach in Chapter 9 of [8]. For some linear systems, the approach shows that the process is survival when the second moments are uniformly bounded.

As a tool, we introduce a stochastic process $\zeta_{t}$ which is a modification of $\xi_{t}$ introduced in Section 3. For $\zeta_{t}$ on $\mathbf{Z}^{d}$, the state space of $\zeta_{t}$ is $[0,+\infty)^{\mathbf{Z}^{d}}$, which means that at each vertex $x \in \mathbf{Z}^{d}$ there is a nonnegative real number. At event times of $N_{x}(\cdot)$ and $Y_{x}(\cdot), \zeta_{t}$ flips in the same way as that of $\xi_{t}$. What different from $\xi_{t}$ is that $\zeta_{t}$ evolves according to an linear ODE between event times of Poisson processes. In detail, for each $x \in \mathbf{Z}^{d}, \zeta_{s}(x)=0$ at event time $s$ of $N_{x}(\cdot)$ while $\zeta_{r}(x)=\zeta_{r-}(x)+\sum_{y: y \sim x} \zeta_{r-}(y)$ at event time $r$ of $Y_{x}(\cdot)$. Between any two adjacent event times of the Poisson processes $N_{x}(\cdot)$ and $Y_{x}(\cdot), \zeta_{t}(x)$ evolves according to the following ODE

$$
\frac{d}{d t} \zeta_{t}(x)=(1-2 \lambda d) \zeta_{t}(x)
$$

The generator of $\zeta_{t}$ is given by

$$
\begin{aligned}
\Omega f(\zeta)= & \sum_{x \in \mathbf{Z}^{d}}\left[f\left(\zeta^{x, 0}\right)-f(\zeta)\right] \\
& +\sum_{x \in \mathbf{Z}^{d}} \lambda\left[f\left(\zeta^{x, \zeta(x)+\sum_{y: y \sim x} \zeta(y)}\right)-f(\zeta)\right]+\sum_{x \in \mathbf{Z}^{d}} f_{x}^{\prime}(\zeta)(1-2 \lambda d) \zeta(x)
\end{aligned}
$$

for any $\zeta \in[0,+\infty)^{\mathbf{Z}^{d}}$ and $f \in C^{1}\left([0,+\infty)^{\mathbf{Z}^{d}}\right)$, where $f_{x}^{\prime}(\zeta)$ is the partial derivative of $f(\zeta)$ with respect to the coordinate $\zeta(x)$ (See Chapter 9 of 8 for more about generator of a linear system.).

The following Lemma shows that uniformly bounded second moments of $\zeta_{t}$ ensure the survival of $\eta_{t}$, which is crucial for our approach.

Lemma 5.1. If $\lambda$ makes

$$
\sup _{t \geq 0} E\left(\zeta_{t}^{\delta_{1}}(x)\right)^{2}<+\infty
$$

for each $x \in Z^{d}$, then $\lambda_{c}^{Z^{d}} \leq \lambda$.

Notice that $E\left(\zeta_{t}^{\delta_{1}}(x)\right)^{2}$ does not depending on $x$ since $\mathbf{Z}^{d}$ is symmetric. 
Proof of Lemma 5.1. For each $x \in \mathbf{Z}^{d}$ and $t \geq 0$, let $\widetilde{\eta}_{t}(x)=1_{\left\{\zeta_{t}(x)>0\right\}}$. After a similar discussion with that of $\xi_{t}$, it is easy to see that $\widetilde{\eta}_{t}$ is also a threshold-one contact process with flip rates given by (1.1). Therefore, $\eta_{t}^{\delta_{1}}$ and $\zeta_{t}^{\delta_{1}}$ with same infection rate $\lambda$ on $\mathbf{Z}^{d}$ can be coupled such that

$$
\eta_{t}^{\delta_{1}}(x)=1_{\left\{\zeta_{t}^{\delta_{1}}(x)>0\right\}}
$$

for each $x \in \mathbf{Z}^{d}$. Then by Hölder inequality,

$$
P\left(\eta_{t}^{\delta_{1}}(x)=1\right)=P\left(\zeta_{t}^{\delta_{1}}(x)>0\right) \geq \frac{\left(E \zeta_{t}^{\delta_{1}}(x)\right)^{2}}{E\left(\zeta_{t}^{\delta_{1}}(x)\right)^{2}} .
$$

According to the generator of $\zeta_{t}$ given in (5.1) and a similar proof with that of (3.6) (See Appendix A.2),

$$
\frac{d}{d t} E \zeta_{t}^{\delta_{1}}(x)=-E \zeta_{t}^{\delta_{1}}(x)+\lambda \sum_{y: y \sim x} E \zeta_{t}^{\delta_{1}}(y)+(1-2 \lambda d) E \zeta_{t}^{\delta_{1}}(x)
$$

for each $x \in \mathbf{Z}^{d}$. Due to the symmetry of $\mathbf{Z}^{d}, E \zeta_{t}^{\delta_{1}}(x)$ does not depending on $x$. Therefore,

$$
\frac{d}{d t} E \zeta_{t}^{\delta_{1}}(x)=-E \zeta_{t}^{\delta_{1}}(x)+2 d \lambda E \zeta_{t}^{\delta_{1}}(x)+(1-2 \lambda d) E \zeta_{t}^{\delta_{1}}(x)=0
$$

and

$$
E \zeta_{t}^{\delta_{1}}(x) \equiv E \zeta_{0}^{\delta_{1}}(x)=1
$$

for any $t \geq 0$. Therefore, $P\left(\eta_{t}^{\delta_{1}}(x)=1\right) \geq 1 / E\left(\zeta_{t}^{\delta_{1}}(x)\right)^{2}$. If $\lambda$ makes

$$
\sup _{t \geq 0} E\left(\zeta_{t}^{\delta_{1}}(x)\right)^{2}<+\infty
$$

then

$$
\mu_{\lambda}^{\mathbf{Z}^{d}}(x)=\lim _{t \rightarrow+\infty} P\left(\eta_{t}^{\delta_{1}}(x)=1\right) \geq 1 / \sup _{t \geq 0} E\left(\zeta_{t}^{\delta_{1}}(x)\right)^{2}>0
$$

and hence $\lambda \geq \lambda_{c}^{\mathbf{Z}^{d}}$.

Now the main problem is to find $\lambda$ making $\sup _{t \geq 0} E\left(\zeta_{t}^{\delta_{1}}(x)\right)^{2}<+\infty$. First we give the ODE which $\left\{E\left(\zeta_{t}^{\delta_{1}}(x)\right)^{2}\right\}_{x \in \mathbf{Z}^{d}}$ satisfying. By the symmetry of $\mathbf{Z}^{d}$, we define

$$
G_{t}(x)=E\left[\zeta_{t}^{\delta_{1}}(0) \zeta_{t}^{\delta_{1}}(x)\right]=E\left[\zeta_{t}^{\delta_{1}}(y) \zeta_{t}^{\delta_{1}}(x+y)\right]
$$


for any $x, y \in \mathbf{Z}^{d}$ and $t \geq 0$. Then $E\left(\zeta_{t}^{\delta_{1}}(x)\right)^{2}=G_{t}(0)$. According to the generator of $\zeta_{t}$, we can show that $\left\{G_{t}(x)\right\}_{x \in \mathbf{Z}^{d}}$ satisfies

$$
\frac{d}{d t} G_{t}(x)=4 \lambda d\left[\frac{1}{2 d} \sum_{y: y \sim x} G_{t}(y)-G_{t}(x)\right]
$$

for any $x \neq 0$ and

$$
\begin{aligned}
\frac{d}{d t} G_{t}(0) & =(1-4 \lambda d) G_{t}(0)+2 \lambda \sum_{y: y \sim 0} G_{t}(y)+\lambda \sum_{y: y \sim 0} \sum_{z: z \sim 0} G_{t}(y+z) \\
& =(1-2 \lambda d) G_{t}(0)+2 \lambda \sum_{y: y \sim 0} G_{t}(y)+\lambda \sum_{y: y \sim 0} \sum_{\substack{z: z \sim 0, z \neq-y}} G_{t}(y+z) .
\end{aligned}
$$

In other words,

$$
\frac{d}{d t} G_{t}=Q G_{t}
$$

where $Q$ is a $\mathbf{Z}^{d} * \mathbf{Z}^{d}$ matrix such that

$$
Q\left(x_{1}, x_{2}\right)= \begin{cases}-4 \lambda d & \text { if } x_{1}=x_{2} \neq 0 \\ 2 \lambda & \text { if } x_{1} \neq 0, x_{2} \sim x_{1} \\ 1-2 \lambda d & \text { if } x_{1}=x_{2}=0 \\ 2 \lambda & \text { if } x_{1}=0, x_{2} \sim 0 \\ \sum_{\substack{(y, z): y \sim 0 \\ z \sim 0, y+z=x_{2}}} \lambda & \text { if } x_{1}=0,\left\|x_{2}\right\|=2 \\ 0 & \text { else }\end{cases}
$$

for $x_{1}, x_{2} \in \mathbf{Z}^{d}$.

(5.4) and (5.5) are also with the form $\frac{d}{d t} E f\left(\zeta_{t}\right)=E \Omega f\left(\zeta_{t}\right)$ as (3.6). To prove these two equations rigorously, we need Theorem 3.1 of Chapter 9 of [8]. For more details, see Appendix A.2.

The following Lemma gives a sufficient condition for $\sup _{t \geq 0} E\left(\zeta_{t}^{\delta_{1}}(x)\right)^{2}<$ $+\infty$.

Lemma 5.2. If there exists a function $h: Z^{d} \rightarrow \boldsymbol{R}$ such that

$$
0<\inf _{x \in Z^{d}} h(x) \leq \sup _{x \in Z^{d}} h(x)<+\infty
$$

and

$$
Q h=0,
$$

then

$$
\sup _{t \geq 0} E\left(\zeta_{t}^{\delta_{1}}(x)\right)^{2} \leq \frac{\sup _{x \in Z^{d}} h(x)}{\inf _{x \in Z^{d}} h(x)}<+\infty .
$$


The following proof of Lemma 5.2 need several characters of the matrix $Q$. We will prove these characters rigorously in Appendix A.3.

Proof. In Theorem A.1 of Appendix A.3 we will show that

$$
\left|Q^{n}(x, y)\right| \leq\left(1+8 \lambda d+4 \lambda d^{2}\right)^{n}
$$

for any $x, y \in \mathbf{Z}^{d}$.

Therefore it is reasonable to define

$$
\exp \{t Q\}=\sum_{n=0}^{+\infty} \frac{(t Q)^{n}}{n !}
$$

for any $t \geq 0$.

We denote by $L^{\infty}\left(\mathbf{Z}^{d}\right)$ the set of bounded functions on $\mathbf{Z}^{d}$ and define

$$
\|f\|_{\infty}=\sup _{x \in \mathbf{Z}^{d}}|f(x)|
$$

for $f \in L^{\infty}\left(\mathbf{Z}^{d}\right)$.

According to classical theorems of linear ODE, we will show in Theorem A.2 of Appendix A.3 that the unique solution to the following equation

$$
\frac{d}{d t} f_{t}=Q f_{t}
$$

with initial condition $f_{0} \in L^{\infty}\left(\mathbf{Z}^{d}\right)$ is

$$
f_{t}=\exp \{t Q\} f_{0}
$$

and $\left\|f_{t}\right\|_{\infty} \leq \exp \left\{t\left(1+8 \lambda d+4 \lambda d^{2}\right)\right\}\left\|f_{0}\right\|_{\infty}$.

As a result,

$$
\begin{aligned}
E\left(\zeta_{t}^{\delta_{1}}(0)\right)^{2} & =G_{t}(0)=\sum_{x \in \mathbf{Z}^{d}} \exp \{t Q\}(0, x) G_{0}(x) \\
& =\sum_{x \in \mathbf{Z}^{d}} \exp \{t Q\}(0, x) E\left(\zeta_{0}^{\delta_{1}}(0) \zeta_{0}^{\delta_{1}}(x)\right)=\sum_{x \in \mathbf{Z}^{d}} \exp \{t Q\}(0, x) .
\end{aligned}
$$

According to the definition of $\exp \{t Q\}$ and Fubini Theorem,

$$
Q \exp \{t Q\}=\sum_{n=0}^{+\infty} \frac{t^{n} Q^{n+1}}{n !}=\exp \{t Q\} Q
$$

Since $Q h=0$,

$$
\frac{d}{d t} \exp \{t Q\} h=Q \exp \{t Q\} h=\exp \{t Q\} Q h=0
$$


and $\exp \{t Q\} h \equiv h$ for $t \geq 0$. Therefore,

$$
h(0)=\exp \{t Q\} h(0)=\sum_{x \in \mathbf{Z}^{d}} \exp \{t Q\}(0, x) h(x)
$$

for $t \geq 0$. In Theorem A.3 of Appendix $\mathrm{A.3}$ we will show that

$$
\exp \{t Q\}(x, y) \geq 0
$$

for any $(x, y) \in \mathbf{Z}^{d}$. Then

$$
\begin{aligned}
E\left(\zeta_{t}^{\delta_{1}}(0)\right)^{2} & =\sum_{x \in \mathbf{Z}^{d}} \exp \{t Q\}(0, x) \\
& \leq \sum_{x \in \mathbf{Z}^{d}} \exp \{t Q\}(0, x) \frac{h(x)}{\inf _{y \in \mathbf{Z}^{d}} h(y)} \\
& =\frac{h(0)}{\inf _{y \in \mathbf{Z}^{d}} h(y)} \\
& \leq \frac{\sup _{x \in \mathbf{Z}^{d}} h(x)}{\inf _{x \in \mathbf{Z}^{d}} h(x)}
\end{aligned}
$$

for $t \geq 0$ and the proof complete.

To construct $h$ satisfying (5.8), we consider simple random walk $S_{n}^{(d)}$ on $\mathbf{Z}^{d}$. Let $\tau_{0}^{(d)}=\inf \left\{m \geq 1: S_{m}^{(d)}=0\right\}$. For $d \geq 1$, we define $F_{d}: \mathbf{Z}^{d} \rightarrow \mathbf{R}$ as

$$
F_{d}(x)=P\left(\tau_{0}^{(d)}<+\infty \mid S_{0}=x\right)
$$

for $x \in Z^{d} \backslash\{0\}$ and $F_{d}(0)=1$. Let $e_{1}^{(d)}=(1, \underbrace{0,0, \ldots, 0}_{d-1 \mathrm{th}})$, then the following estimation of $F_{d}\left(e_{1}^{(d)}\right)$ is crucial for us to construct $h$.

\section{Lemma 5.3.}

$$
\lim _{d \rightarrow+\infty} 2 d F_{d}\left(e_{1}^{(d)}\right)=1 .
$$

We do not know whether (5.10) has been proven in early references about simple random walk. We searched several famous books such as [7] and 13] but can not find this conclusion, so we give our own proof of (5.10) in Appendix A.1

By (5.10),

$$
\frac{1}{4 d\left[1-(d+1) F_{d}\left(e_{1}^{(d)}\right)\right]}>0
$$

for sufficient large $d$. Finally we can construct $h$ and give an upper bound of $\lambda_{c}^{\mathbf{Z}^{d}}$. 
Theorem 5.4. For sufficient large d such that $\frac{1}{4 d\left[1-(d+1) F_{d}\left(e_{1}^{(d)}\right)\right]}>0$ and

$$
\lambda>\frac{1}{4 d\left[1-(d+1) F_{d}\left(e_{1}^{(d)}\right)\right]},
$$

we define

$$
b_{\lambda}=\frac{4 d \lambda\left[1-(d+1) F_{d}\left(e_{1}^{(d)}\right)\right]-1}{1+4 d^{2} \lambda}
$$

and

$$
h(x)=F_{d}(x)+b_{\lambda}
$$

for each $x \in Z^{d}$. Then $h$ satisfies (5.7) and (5.8). As a result,

$$
\lambda_{c}^{Z^{d}} \leq \frac{1}{4 d\left[1-(d+1) F_{d}\left(e_{1}^{(d)}\right)\right]} .
$$

and

$$
\limsup _{t \rightarrow+\infty} 2 d \lambda_{c}^{Z^{d}} \leq 1
$$

Proof. When $\lambda>\frac{1}{4 d\left[1-(d+1) F_{d}\left(e_{1}^{(d)}\right)\right]}$,

$$
0<b_{\lambda} \leq \inf h(x) \leq \sup h(x) \leq 1+b_{\lambda}<+\infty \text {. }
$$

Hence $h$ satisfies (5.7). For (5.8), when $x \neq 0$,

$$
4 \lambda d\left[\frac{1}{2 d} \sum_{y: y \sim x} h(y)-h(x)\right]=4 \lambda d\left[\frac{1}{2 d} \sum_{y: y \sim x} F_{d}(y)-F_{d}(x)\right]=0
$$

according to the probability transition of $S_{n}$. For the case of 0 ,

$$
\begin{aligned}
& (1-4 \lambda d) h(0)+2 \lambda \sum_{y: y \sim 0} h(y)+\lambda \sum_{y: y \sim 0} \sum_{z: z \sim 0} h(y+z) \\
= & \left(1+4 \lambda d^{2}\right) b_{\lambda}+(1-4 \lambda d)+2 \lambda \sum_{y: y \sim 0} F_{d}(y)+\lambda \sum_{y: y \sim 0}\left[1+\sum_{\substack{z: z \sim 0, z \neq-y}} F_{d}(y+z)\right] \\
= & \left(1+4 \lambda d^{2}\right) b_{\lambda}+(1-4 \lambda d)+2 \lambda \sum_{y: y \sim 0} F_{d}(y)+\lambda \sum_{y: y \sim 0} 2 d F_{d}(y) \\
= & \left(1+4 \lambda d^{2}\right) b_{\lambda}+(1-4 \lambda d)+4 \lambda d(d+1) F_{d}\left(e_{1}^{(d)}\right) \\
= & \left(1+4 \lambda d^{2}\right) b_{\lambda}+1-4 \lambda d\left[1-(d+1) F_{d}\left(e_{1}^{(d)}\right)\right] \\
= & 0
\end{aligned}
$$


according to the definition of $b_{\lambda}$ and $h$. Notice that during the calculation, we use that $F_{d}(y)=F_{d}\left(e_{1}^{(d)}\right)$ for $y \sim 0$ since $\mathbf{Z}^{d}$ is symmetric and

$$
\frac{1}{2 d}+\frac{1}{2 d} \sum_{\substack{z: z \sim 0, z \neq-y}} F_{d}(y+z)=F_{d}(y)
$$

for $y \sim 0$.

The calculation above shows that $Q h=0$. By Lemma 5.1 and Lemma 5.2

$$
\lambda \geq \lambda_{c}^{\mathbf{Z}^{d}}
$$

for any $\lambda>\frac{1}{4 d\left[1-(d+1) F_{d}\left(e_{1}^{(d)}\right)\right]}$ and hence $\lambda_{c}^{\mathbf{Z}^{d}} \leq \frac{1}{4 d\left[1-(d+1) F_{d}\left(e_{1}^{(d)}\right)\right]}$. Furthermore, $\lim \sup _{t \rightarrow+\infty} 2 d \lambda_{c}^{\mathbf{Z}^{d}} \leq 1$ holds by (5.10).

(2.1) is a direct corollary of Corollary 3.2 and Theorem 5.4 For large $d$, we shows that

$$
\frac{1}{2 d} \leq \lambda_{c}^{\mathbf{Z}^{d}} \leq \frac{1}{4 d\left[1-(d+1) F_{d}\left(e_{1}^{(d)}\right)\right]} .
$$

Now the whole proof of Theorem 2.1 is accomplished.

\section{A Appendix}

\section{A.1 Proof of (5.10)}

Proof of (5.10). According to classical theory of simple random walk (See 7 ] and [13].),

$$
F_{d}\left(e_{1}^{(d)}\right)=\frac{G_{d}(0,0)-1}{G_{d}(0,0)}
$$

where $S_{n}^{(d)}$ is simple random walk on $\mathbf{Z}^{d}$ with $S_{0}^{(d)}=0$ and

$$
G_{d}(0,0)=1+\sum_{n=1}^{+\infty} P\left(S_{2 n}^{(d)}=0\right) .
$$

Hence we only need to show that $\lim _{d \rightarrow+\infty} 2 d\left[G_{d}(0,0)-1\right]=1$.

$$
G_{d}(0,0)-1=2 d\left(\frac{1}{2 d}\right)^{2}+\sum_{n=2}^{+\infty} P\left(S_{2 n}^{(d)}=0\right)=\frac{1}{2 d}+\sum_{n=2}^{+\infty} P\left(S_{2 n}^{(d)}=0\right) .
$$

Hence we only need to show that $\lim _{d \rightarrow+\infty} d \sum_{n=2}^{+\infty} P\left(S_{2 n}^{(d)}=0\right)=0$. Let

$$
H_{d}(1)=\sum_{n=2}^{d} P\left(S_{2 n}^{(d)}=0\right)
$$


and

$$
H_{d}(k)=\sum_{n=(k-1) d+1}^{k d} P\left(S_{2 n}^{(d)}=0\right)
$$

for $k \geq 2$. Then $\sum_{n=2}^{+\infty} P\left(S_{2 n}^{(d)}=0\right)=\sum_{k=1}^{+\infty} H_{d}(k)$.

$$
\begin{aligned}
H_{d}(1) & \leq \sum_{n=2}^{d}\left(\begin{array}{c}
2 n \\
n
\end{array}\right) d^{n} n !\left(\frac{1}{2 d}\right)^{2 n} \\
& =\sum_{n=2}^{d} L(n, d)
\end{aligned}
$$

where

$$
L(n, d)=\frac{(2 n-1) ! !}{(2 d)^{n}} .
$$

$L(n, d)=L(n-1, d) \frac{2 n-1}{2 d}$, hence $L(n, d)$ decreases with $n$ when $n<\lceil d\rceil$ and increases with $n$ when $n \geq\lceil d\rceil$. When $n \leq \frac{d}{2}, L(n, d) \leq \frac{1}{2} L(n-1, d)$ and $L(n, d) \leq \frac{1}{2^{n-2}} L(2, d)$. Therefore,

$$
\begin{aligned}
H_{d}(1) & \leq L(2, d) \sum_{n=2}^{\left\lfloor\frac{d}{2}\right\rfloor} \frac{1}{2^{n-2}}+\left(d-\left\lfloor\frac{d}{2}\right\rfloor\right) L\left(\left\lfloor\frac{d}{2}\right\rfloor, d\right) \\
& \leq \frac{3}{2 d^{2}}+d L\left(\left\lfloor\frac{d}{2}\right\rfloor, d\right) .
\end{aligned}
$$

By Stirling formula,

$$
\begin{aligned}
L\left(\left\lfloor\frac{d}{2}\right\rfloor, d\right) & =\frac{\left(2\left\lfloor\frac{d}{2}\right\rfloor\right) !}{(2 d)^{\left\lfloor\frac{d}{2}\right\rfloor}\left\lfloor\frac{d}{2}\right\rfloor ! 2^{\left\lfloor\frac{d}{2}\right\rfloor}} \\
& =\frac{\sqrt{2(1+o(1))}}{(2 e)^{\left\lfloor\frac{d}{2}\right\rfloor}} .
\end{aligned}
$$

Therefore,

$$
H_{d}(1) \leq \frac{3}{2 d^{2}}+\frac{2 d}{(2 e)^{\left\lfloor\frac{d}{2}\right\rfloor}}
$$

for sufficiently large $d$ and $\lim _{d \rightarrow+\infty} d H_{d}(1)=0$

Since $L(n, d)$ increases with $n$ when $n \geq d+1$,

$$
\begin{aligned}
H_{d}(2) & \leq \sum_{n=d+1}^{2 d} L(n, d) \\
& \leq d L(2 d, d) \\
& =d \sqrt{2(1+o(1))}\left(\frac{2}{e}\right)^{2 d}
\end{aligned}
$$


by Stirling formula. Therefore $H_{d}(2) \leq 2 d\left(\frac{2}{e}\right)^{2 d}$ for sufficiently large $d$ and $\lim _{d \rightarrow+\infty} d H_{d}(2)=0$.

Finally we will show that $\lim _{d \rightarrow+\infty} d \sum_{k=3}^{+\infty} H_{d}(k)=0$. For $n \geq 1$, we define

$$
\beta(n)=\frac{n !}{\sqrt{2 \pi n}\left(\frac{n}{e}\right)^{n}} .
$$

By Stirling formula, $\lim _{n \rightarrow+\infty} \beta(n)=1$. Hence there exists $N_{1}$ such that $\frac{\beta_{2 n}}{\beta_{n}}<$ 2 for any $n \geq N_{1}$. For $1 \leq j \leq d$ and $k \geq 3$,

$$
\begin{aligned}
P\left(S_{2(k d+j)}^{(d)}=0\right) & =\sum_{l_{1}+l_{2}+\ldots+l_{d}=k d+j} \frac{[2(k d+j)] !}{\left(l_{1} !\right)^{2}\left(l_{2} !\right)^{2} \ldots\left(l_{d} !\right)^{2}}\left(\frac{1}{2 d}\right)^{2(k d+j)} \\
& =\frac{[2(k d+j)] !}{(k d+j) !(k d+j) !} \sum_{l_{1}+l_{2}+\ldots+l_{d}=k d+j}\left[\frac{(k d+j) !}{l_{1} ! l_{2} ! \ldots l_{d} !}\right]^{2}\left(\frac{1}{2 d}\right)^{2(k d+j)}
\end{aligned}
$$

Since $l_{1} ! l_{2} ! \ldots l_{d} ! \geq(k !)^{d-j}((k+1) !)^{j}$ and

$$
\sum_{l_{1}+l_{2}+\ldots+l_{d}=k d+j} \frac{(k d+j) !}{l_{1} ! l_{2} ! \ldots l_{d} !}=d^{k d+j},
$$

we have

$$
\begin{aligned}
& P\left(S_{2(k d+j)}^{(d)}=0\right) \\
& \leq \frac{\beta(2(k d+j)) 2^{2(k d+j)}}{\beta^{2}(k d+j)} \sqrt{\frac{1}{\pi(k d+j)}} \frac{(k d+j) ! d^{k d+j}}{(k !)^{d-j}((k+1) !)^{j}}\left(\frac{1}{2 d}\right)^{2(k d+j)} \\
& =\sqrt{2} \frac{\beta(2(k d+j))}{\beta(k d+j)} \frac{\left(k+\frac{j}{d}\right)^{k d+j}}{e^{k d+j}(k !)^{d-j}((k+1) !)^{j}} \\
& \leq \sqrt{2} \frac{\beta(2(k d+j))}{\beta(k d+j)}\left[\frac{(k+1)^{k}}{e^{k} k !}\right]^{d} .
\end{aligned}
$$

Let $M_{k}=\frac{(k+1)^{k}}{e^{k} k !}$, then $\frac{M_{k+1}}{M_{k}}=\left(1+\frac{1}{k+1}\right)^{k+1} / e<1$, and hence $\sup _{k \geq 2} M_{k}=$ $M_{2}=\frac{9}{2 e^{2}}<1$. By Stirling Formula, $\lim _{k \rightarrow+\infty} \sqrt{2 \pi k} M_{k}=e$ and hence $C=$ $\sup _{k \geq 2} \sqrt{k} M_{k}<+\infty$. Choose $N_{2}$ such that $\frac{C}{\sqrt{N_{2}}}<\frac{1}{2}$, then for $d>N_{1}$,

$$
\begin{aligned}
\sum_{k=3}^{+\infty} H_{d}(k) & \leq \sum_{k=3}^{+\infty} 2 \sqrt{2} d M_{k-1}^{d} \\
& \leq 2 \sqrt{2} d N_{2} M_{2}^{d}+2 \sqrt{2} d C^{d} \sum_{m=N_{2}+1}^{+\infty} k^{-\frac{d}{2}} \\
& \leq 2 \sqrt{2} d N_{2} M_{2}^{d}+2 \sqrt{2} d C^{d} \int_{N_{2}}^{+\infty} x^{-\frac{d}{2}} d x \\
& =2 \sqrt{2} d N_{2} M_{2}^{d}+\frac{4 \sqrt{2} d N_{2}}{d-2}\left(\frac{C}{\sqrt{N_{2}}}\right)^{d},
\end{aligned}
$$


and

$$
d \sum_{k=3}^{+\infty} H_{d}(k) \leq 2 \sqrt{2} d^{2} N_{2} M_{2}^{d}+\frac{4 \sqrt{2} d^{2} N_{2}}{d-2}\left(\frac{1}{2}\right)^{d} .
$$

Since $M_{2}<1$,

$$
\lim _{d \rightarrow+\infty} d \sum_{k=3}^{+\infty} H_{d}(k)=0
$$

As a result,

$$
\lim _{d \rightarrow+\infty} d \sum_{n=2}^{+\infty} P\left(S_{2 n}^{(d)}=0\right)=\lim _{d \rightarrow+\infty} d\left(H_{d}(1)+H_{d}(2)+\sum_{k=3}^{+\infty} H_{d}(k)\right)=0 .
$$

\section{A.2 Proof of (3.6), (5.3), (5.4) and (5.5).}

In this subsection we give the rigorous proofs of (3.6), (5.3), (5.4) and (5.5).

Proof of (3.6). For any $t>0$, we define that $\beta_{t}=\xi_{\frac{1}{1+\lambda} t}$. According to the flip-rates of $\xi_{t}, \beta_{t}$ is a standard linear system introduced in Chapter 9.0 of $[8$ ] with

$$
a(u, v)=0
$$

for any $u, v \in G$ and

$$
A_{x}(u, v)= \begin{cases}1 & \text { if } \quad u=v \neq x \\ 0 & \text { else }\end{cases}
$$

with probability $\frac{1}{1+\lambda}$ and

$$
A_{x}(u, v)= \begin{cases}1 \quad & \text { if } \quad u=v \\ 1 \quad & \text { else if } \quad u=x \quad \text { and } \quad v \sim x \\ 0 \quad \text { else } & \end{cases}
$$

with probability $\frac{\lambda}{1+\lambda}$ for any $u, v, x \in G$.

According to Theorem 1.27 of Chapter 9 of 8 ,

$$
\frac{d}{d t} E \beta_{t}^{\delta_{1}}(x)=\sum_{y} \gamma(x, y) E \beta_{t}^{\delta_{1}}(y)
$$


where

$$
\gamma(x, y)=a(x, y)+ \begin{cases}\mathrm{E} \sum_{u} A_{u}(x, y) & \text { if } x \neq y, \\ \mathrm{E} \sum_{u}\left[A_{u}(x, x)-1\right] & \text { else. }\end{cases}
$$

By the definition of $a(\cdot, \cdot),\left\{A_{x}(\cdot, \cdot)\right\}_{x \in G}$ and direct calculation,

$$
\gamma(x, y)= \begin{cases}-\frac{1}{1+\lambda} & \text { if } \quad x=y \\ \frac{\lambda}{1+\lambda} & \text { else if } \quad y \sim x \\ 0 & \text { else }\end{cases}
$$

and

$$
\frac{d}{d t} E \beta_{t}^{\delta_{1}}(x)=-\frac{1}{1+\lambda} E \beta_{t}^{\delta_{1}}(x)+\sum_{y: y \sim x} \frac{\lambda}{1+\lambda} E \beta_{t}^{\delta_{1}}(y) .
$$

Since $E \xi_{t}^{\delta_{1}}(x)=E \beta_{(1+\lambda) t}^{\delta_{1}}(x)$ for any $x \in G$, (3.6) is a direct corollary of (A.8).

Proof of (5.3). For any $t>0$, we define that $\alpha_{t}=\zeta_{\frac{1}{1+\lambda} t}$. Then $\alpha_{t}$ is a standard linear model with

$$
a(x, y)= \begin{cases}\frac{1-2 d \lambda}{1+\lambda} & \text { if } x=y, \\ 0 & \text { else }\end{cases}
$$

for any $x, y \in \mathbf{Z}^{d}$ and the same $\left\{A_{x}(\cdot, \cdot)\right\}_{x \in \mathbf{Z}^{d}}$ as that of $\beta_{t}$ in the proof of (3.6). As we have done in the proof of (3.6), we can obtain (5.3) by directly applying Theorem 1.27 of Chapter 9 of 8 . We omit the details.

Proof of (5.4) and (5.5).$\alpha_{t}$ is the same as that in the proof of (5.3). We use $g(t, x, y)$ to denote $E \alpha_{t}^{\delta_{1}}(x) \alpha_{t}^{\delta_{1}}(y)$ for any $t \geq 0$ and $x, y \in \mathbf{Z}^{d} .\left\{A_{x}(\cdot, \cdot)\right\}_{x \in \mathbf{Z}^{d}}$ and $a(\cdot, \cdot)$ are the same as that in the proof of (5.3). By direct calculation it is easy to verify that

$$
\mathrm{E} \sum_{x \in \mathbf{Z}^{d}}\left[\left|A_{x}(u, u)-1\right|+\sum_{v: v \neq u} A_{x}(u, v)\right]^{2}<+\infty
$$

for any $u \in \mathbf{Z}^{d}$.

Then according to Theorem 3.1 of Chapter 9 of $[\underline{8}, g(t, x, y)$ satisfies that $g(0, x, y)=1$ and

$$
\frac{d}{d t} g(t, x, y)=\sum_{u, v \in \mathbf{Z}^{d}} q((x, y),(u, v)) g(t, u, v)
$$


where

$$
q((x, y),(u, v))= \begin{cases}\mathrm{E} \sum_{z} A_{z}(x, u) A_{z}(y, v) & \text { if } u \neq x, v \neq y, \\ \mathrm{E} \sum_{z} A_{z}(x, x) A_{z}(y, v)+a(y, v) & \text { else if } u=x, v \neq y, \\ \mathrm{E} \sum_{z} A_{z}(x, u) A_{z}(y, y)+a(x, u) & \text { else if } u \neq x, v \neq y, \\ \mathrm{E} \sum_{z}\left[A_{z}(x, x) A_{z}(y, y)-1\right]+a(x, x)+a(y, y) & \text { else. }\end{cases}
$$

According to the definition of $\left\{A_{x}(\cdot, \cdot)\right\}_{x \in \mathbf{Z}^{d}}$ and $a(\cdot, \cdot)$ of $\alpha_{t}$,

$$
q((x, x),(u, v))=\left\{\begin{array}{lll}
\frac{1-4 \lambda d}{1+\lambda} & \text { if } \quad u=x, v=x, \\
\frac{\lambda}{1+\lambda} & \text { else if } \quad u=x, v \sim x, \\
\frac{\lambda}{1+\lambda} & \text { else if } u \sim x, v=x, \\
\frac{\lambda}{1+\lambda} & \text { else if } u \sim x, v \sim x, \\
0 & \text { else }
\end{array}\right.
$$

and

$$
q((x, y),(u, v))= \begin{cases}-\frac{4 \lambda d}{1+\lambda} & \text { if } \quad u=x, v=y \\ \frac{\lambda}{1+\lambda} & \text { else if } \quad u=x, v \sim y \\ \frac{\lambda}{1+\lambda} & \text { else if } u \sim x, v=y \\ 0 & \text { else }\end{cases}
$$

for $x \neq y$.

Since $G_{t}(x)=g((1+\lambda) t, 0, x)=g((1+\lambda) t, y, y+x)$ for any $x, y \in \mathbf{Z}^{d}$, (5.4) and (5.5) are direct corollaries of (A.9), A.10) and A.11).

\section{A.3 Characters of $Q$}

Theorem A.1. $Q$ is the same as that in (5.6). For any $x, y \in \mathbb{Z}^{d}$,

$$
\left|Q^{n}(x, y)\right| \leq\left(1+8 \lambda d+4 \lambda d^{2}\right)^{n} .
$$

Proof. By (5.6) and direct calculation it is easy to see that (A.12) holds for 
$n=1$. When A.12 holds for some $n \geq 1$,

$$
\begin{aligned}
\left|Q^{n+1}(x, y)\right| & =\left|Q Q^{n}(x, y)\right| \\
& =\left|4 \lambda d\left[\frac{1}{2 d} \sum_{z: z \sim x} Q^{n}(z, y)-Q^{n}(x, y)\right]\right| \\
& \leq 8 \lambda d\left(1+8 \lambda d+4 \lambda d^{2}\right)^{n} \\
& \leq\left(1+8 \lambda d+4 \lambda d^{2}\right)^{n+1}
\end{aligned}
$$

for $x \neq 0$ and

$$
\begin{aligned}
\left|Q^{n+1}(0, y)\right| & =\left|Q Q^{n}(0, y)\right| \\
& =\mid(1-4 \lambda d) Q^{n}(0, y)+2 \lambda \sum_{z: z \sim 0} Q^{n}(z, y) \\
& +\lambda \sum_{z: z \sim 0} \sum_{w: w \sim 0} Q(w+z, y) \mid \\
& \leq\left(1+4 \lambda d+4 \lambda d+4 \lambda d^{2}\right)\left(1+8 \lambda d+4 \lambda d^{2}\right)^{n} \\
& =\left(1+8 \lambda d+4 \lambda d^{2}\right)^{n+1} .
\end{aligned}
$$

Therefore A.12 holds for any $n \geq 1$ by induction.

Theorem A.2. $Q$ is the same as that in (5.6). There exists a unique solution $\left\{f_{t} \in L^{\infty}\left(\boldsymbol{Z}^{d}\right), t \geq 0\right\}$ to the following $O D E$

$$
\frac{d}{d t} f_{t}=Q f_{t}
$$

with initial condition that $f_{0} \in L^{\infty}\left(\boldsymbol{Z}^{d}\right)$. Moreover, $f_{t}$ is with the form

$$
f_{t}=\exp \{t Q\} f_{0}
$$

and satisfies

$$
\left\|f_{t}\right\|_{\infty} \leq \exp \left\{1+8 \lambda d+4 \lambda d^{2}\right\}\left\|f_{0}\right\|_{\infty}
$$

for any $t \geq 0$.

Proof. By a calculation similar with that in the proof of Theorem A.1, it is easy to verify that

$$
\|Q f\|_{\infty} \leq\left(1+8 \lambda d+4 \lambda d^{2}\right)\|f\|_{\infty}
$$

for any $f \in L^{\infty}\left(\mathbf{Z}^{d}\right)$. Therefore the linear operator $T: f \rightarrow Q f$ on $L^{\infty}\left(\mathbf{Z}^{d}\right)$ with norm $\|\cdot\|_{\infty}$ satisfies the Lipshitz condition. Then Theorem A.2 follows the classical theory of linear ODE on Banach spaces. 
Theorem A.3. $\exp \{t Q\}(x, y) \geq 0$ for any $x, y \in \boldsymbol{Z}^{d}$.

Proof. We denote by $I_{\mathbf{Z}^{d}}$ the identity matrix $\{\delta(x, y)\}_{x, y \in \mathbf{Z}^{d}}$. Let

$$
B=Q+4 \lambda d I_{\mathbf{Z}^{d}}
$$

then $B(x, y) \geq 0$ for any $x, y \in \mathbf{Z}^{d}$ according to the definition of $Q$. As a result, $\exp \{t B\}(x, y) \geq 0$ for any $x, y \in \mathbf{Z}^{d}$. Since $I_{\mathbf{Z}^{d}} B=B I_{\mathbf{Z}^{d}}$,

$$
\exp \{t Q\}=\exp \{t B\} \exp \left\{-4 t \lambda d I_{\mathbf{Z}^{d}}\right\}
$$

and hence

$$
\exp \{t Q\}(x, y)=\exp \{-4 t \lambda d\} \exp \{t B\}(x, y) \geq 0 .
$$

Acknowledgments. This work is supported by the National Basic Research Program of China (2011CB808000), National Natural Science Foundation of China (No. 11001004) and China Scholarship Council (No. 201206010097).

\section{References}

[1] Andjel, E. D., Liggett, T. M. and Mountford, T. (1992). Clustering in onedimensional threshold voter models. Stochastic Processes and Their Applications 42 73-90.

[2] Cox, J. T. and Durrett, R. (1991). Nonlinear voter models. In Random Walks, Brownian Motion and Interacting Particle Systems. A Festschrift in Honor of Frank Spiter 189-201. Birkhäuser, Boston.

[3] Fontes, L. R., Schonmann, R. H. (2008). Threshold $\theta \geq 2$ contact processes on homogeneous trees. Probability Theory and Related Fields 141: 513-541.

[4] Griffeath, D. (1983). The Binary Contact Path Process. The Annals of Probability 11 692-705.

[5] Handjani, S. (1999). The complete convergence theorem for coexistent threshold voter models. The Annals of Probability 27 226-245.

[6] van der Hofstad, R. (2012). Random Graphs and Complex Networks. Lecture notes, http://www.win.tue.nl/ rhofstad/. 
[7] Lawler, G. F. and Limic, V. (2010) Random Walk: A Modern Introduction. Cambridge University Press.

[8] Liggett, T. M. (1985). Interacting Particle Systems. Springer, New York.

[9] Liggett, T. M. (1994). Coexistence in threshold voter models. The Annals of Probability 22 764-802.

[10] Liggett, T. M. (1999). Stochastic interacting systems: contact, voter and exclusion processes. Springer, New York.

[11] Mountford, T. and Schonmann, R. H. (2009) The survival of large dimensional threshold contact processes. The Annals of Probability 37 1483-1501.

[12] Pemantle, R. (1992). The contact process on trees. The Annals of Probability 20 2089-2116.

[13] Spitzer, F. (1976). Principles of Random Walk. Springer.

[14] Xue, XF. (2012). Critical density points for threshold voter models on homogeneous trees. Journal of Statistical Physics 146 423-433. 